\title{
LENS-INDUCED DISORDERS
}

Phacolytic glaucoma and endophthalmitis phaco-anaphylactica are two lens-induced disorders which are often misdiagnosed clinically, but which present rather specific histopathological pictures. Both conditions can be cured by appropriate surgical intervention.
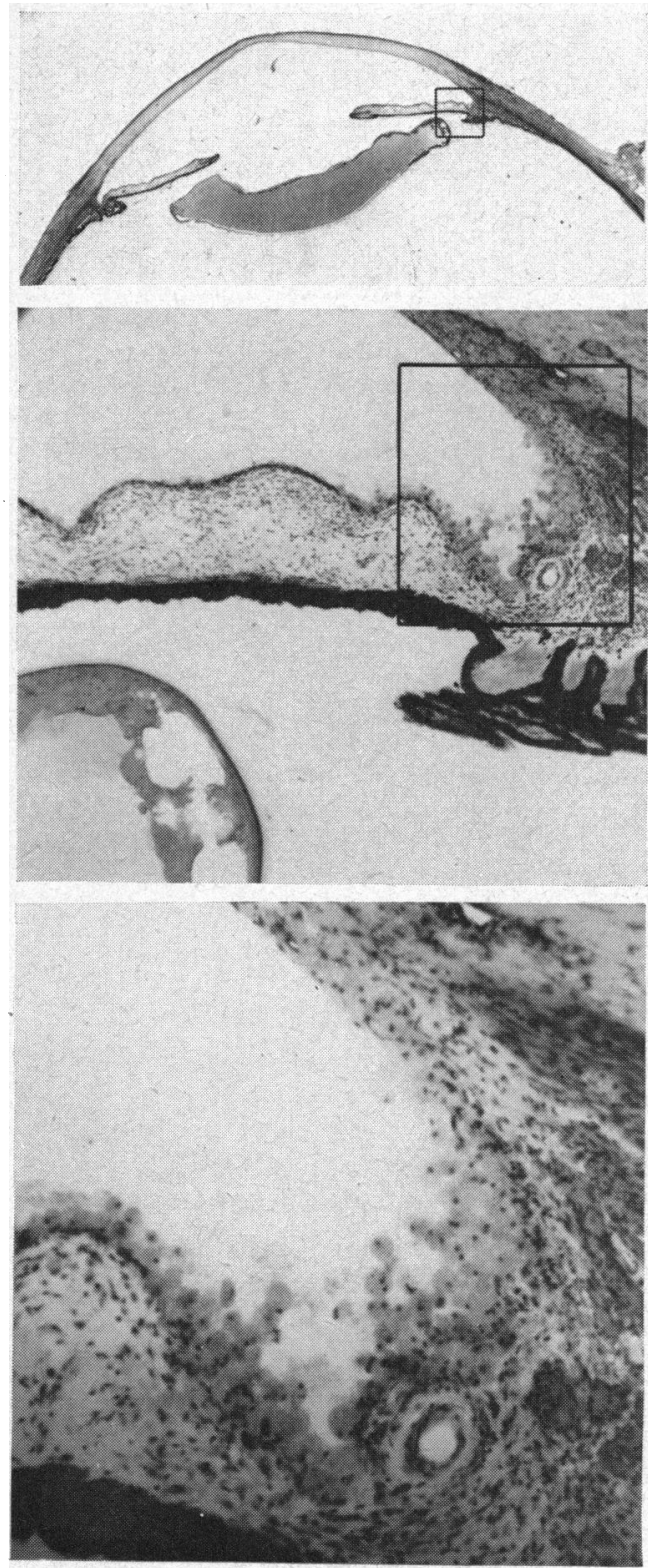

\section{Phacolytic Glaucoma (Fig. 1)}

This condition usually presents as unilateral glaucoma of sudden onset in an older individual with an advanced cataract. In contradistinction to acute closed-angle glaucoma, examination usually reveals a deep anterior chamber with open angles (gonioscopy is vitally important in this and in every case of glaucoma), aqueous flare and cells, and a hypermature cataract.

Pathological examination reveals a hypermature cataract, liquefied cortical material around the lens and within the anterior and posterior chambers, and, most characteristically, swollen macrophages which contain cortical material. These latter cells line the angle of the anterior chamber, and fill the trabecular meshwork and often the iris stroma.

The pathogenesis of this condition is leaking lens material which is phagocytosed by macrophages which then migrate into the anterior chamber where they become trapped in the trabecular meshwork. Glaucoma results from mechanical blockage of the outflow apparatus. Therapy is first directed at lowering the intra-ocular tension, and then removing the offending lens.

FIG. 1.-Phacolytic glaucoma.

Reference:

Flocks, M., Littwin, C. S., and Zimmerman, L. E. (1955). A.M.A. Arch. Ophthal., 54, 37. 


\section{Endophthalmitis Phaco-anaphylactica (Fig. 2)}

An unusual but curable form of uveitis which follows traumatic, operative, or spontaneous rupture of the lens capsule. Usually only the injured eye is affected by the severe inflammation which ensues a few days to several weeks after lens material is liberated into the ocular chambers. Examination reveals severe uveitis with mutton-fat keratic precipitates. Secondary glaucoma may result from synechiae formation.

On histological examination polymorphonuclear leucocytes are found around traumatized or degeneratelensfibres. Surrounding this is a zone of epithelioid cells and granulation tissue. Inflammatory cells including eosinophils are present in the adjacent uvea. The reaction is most marked around the lens.

This condition may result from hypersensitivity to altered lens protein in cataractous lenses. The second eye may be involved, particularly if it is cataractous or if an extracapsular cataract extraction has been performed. When both eyes are involved the condition may resemble sympathetic ophthalmitis, and occasionally both conditions coexist. (Endophthalmitis phaco-anaphylactica usually develops several days after lens extraction or trauma, whereas the onset of sympathetic ophthalmitis is rarely less than two weeks after trauma or operation.) Treatment consists of removing the remaining lens remnants.

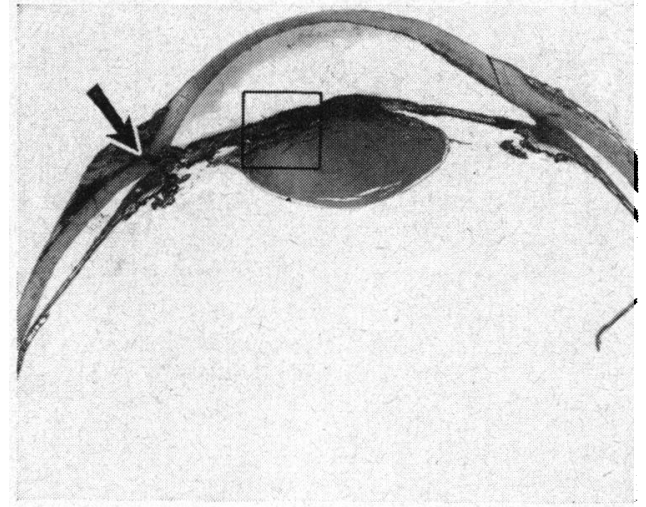

(a)

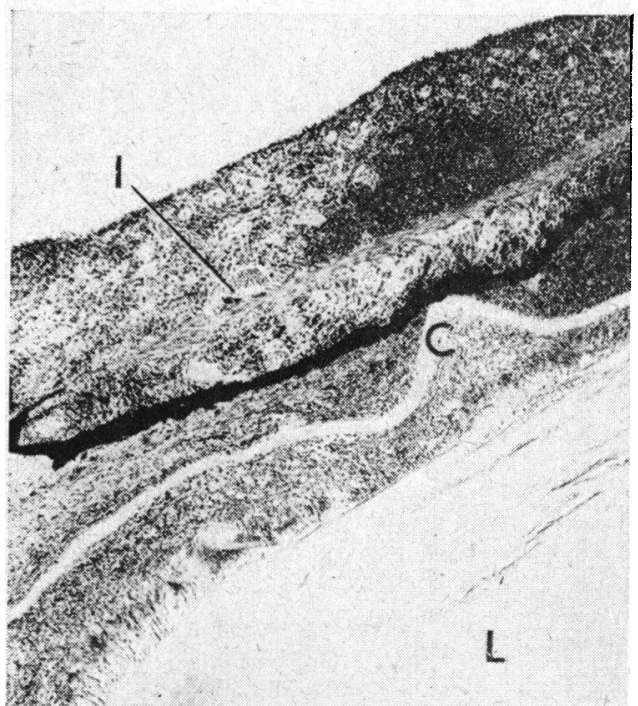

(b)

FIG. 2.-Endophthalmitis phaco-anaphylactica. (a) Low-power view, the arrow pointing to a perforating limbal wound. (b) High-power view, showing iris (I), capsule of lens (C), and lens (L).

\section{Reference:}

Woods, A. C. (1961). "Endogenous Inflammations of the Uveal Tract". Williams and Wilkins, Baltimore.

Illustrations:

INSTITUTE OF OPHTHALMOLOGY
Paul Henkind and Barrie Jay, INSTITUTE OF OPHTHALMOLOGY, LONDON, W.C.1. 